Bundesgesundheitsbl -

Gesundheitsforsch - Gesundheitsschutz

1999 - 42: 436-445 @ Springer-Verlag 1999

Kongressberichte

U. Marcus • Robert Koch-Institut, Berlin

\title{
6. Retroviruskonferenz 1999 in Chicago
}

Teil I

Mehr als 3000 HIV-Expererten aus Praxis und Forschung kamen vom 31. Januar bis 4. Februar 1999 zur 6. Retroviruskonferenz in Chicago zusammen. Die Tagung konzentriert sich in erster Linie auf Fragen der HIV-Grundlagenforschung und Therapieentwicklung, sie gilt neben der Internationalen AIDSKonferenz als bedeutendste internationale, wissenschaftliche Konferenz zu HIV und AIDS. Die wichtigsten Ergebnisse der Konferenz wurden für Sie zusammengefaßt:Teil I des Berichtes informiert vor allem über epidemiologische und Präventionsaspekte sowie über den Stand der Impfdiskussion. Teil II (folgt in Heft 6/99) berichtet über Therapie und Immunrekonstitution.

Die dargestellten Ergebnisse diverser Therapiestudien erlauben einen besseren Vergleich unterschiedlicher Behandlungsstrategien bei Beginn und Wechsel antiretroviraler Therapieregime. Sie deuten darauf hin, daß Resistenzbestimmungen und Medikamentenspiegel-Monitoring eine sinnvolle Rolle bei der Optimierung der Therapie spielen könnten. Neue Daten zu Wechsel- und Nebenwirkungen bzw.zum Nebenwirkungsmanagement ermöglichen eine rationalere Therapieplanung, die sowohl Aspekte der Therapieeffizienz als auch der Lebensqualität berücksichtigt. Die spannendste Entwicklung stellt jedoch die Diskussion um Möglichkeiten und Chancen einer Rekonstitution auch der HIV-spezifischen Immunkompetenz mittels antiretroviraler und immunmodulierender Therapien dar, welche die Hoffnung auf eine Viruseradikation ablöst.Ziel einer solchen Rekonstitution wäre eine langfristige Kontrolle des Immunsystems über die HIV-Infektion ohne Gefahr einer Krankheitsentwicklung, wie sie bei der natürlich vorkommenden SIV-Infektion bei Primaten und eventuell auch bei einer kleinen Zahl von sog. Langzeitinfizierten ohne Krankheitsprogression (LTNP) erfolgt. ie 6. Retroviruskonferenz begann mit einem Blick zurück in die Vergangenheit, genauer gesagt auf die Herkunft des HI-Virus. Nachdem die Entwicklung des zweiten AIDS-Virus HIV-2 bereits vor Jahren schlüssig auf einen wiederholten Spezieswechsel von SIV's von in Westafrika lebenden Halsband-Mangaben auf den Menschen zurückgeführt werden konnte, scheint nunmehr auch die Herkunft von HIV-1 geklärt. Die lang gehegte Vermutung, HIV-1 sei von Schimpansen auf den Menschen übergegangen, wird durch neuere Untersuchungen bestätigt, die letzte noch fehlenden Mosaiksteinchen zur Komplettierung des Bildes beisteuern [1]. Zwar waren die aus Schimpansen isolierten SIVs die dem HIV-1 am nächsten verwandten Immundefizienzviren, aber sie waren genetisch so weit von den HIV-1Subtypen der M und O-Gruppe entfernt, daß sie als direkte Vorfahren schwerlich in Frage kamen. Daher war als alternative Herkunftstherorie bislang nicht auszuschließen, daß ein weiteres und bisher unbekanntes Affen-Immundefizienzvirus entweder direkt oder auf dem Umweg über Schimpansen (die kleinere Affenarten jagen und fressen) der Vorfahre von HIV-1 ist. Untersuchungen von Gewebe einer 1985 verstorbenen Schimpansin mit positivem HIV-Antiköperstatus sowie ein serologisches Screening bei in freier Wildbahn gefangenen Schimpansen in Westafrika.

Die These, daß die HIV-Infektion eine von Affen auf den Menschen übertragene Zoonose ist, stützt sich auf folgende Argumentationslinien:

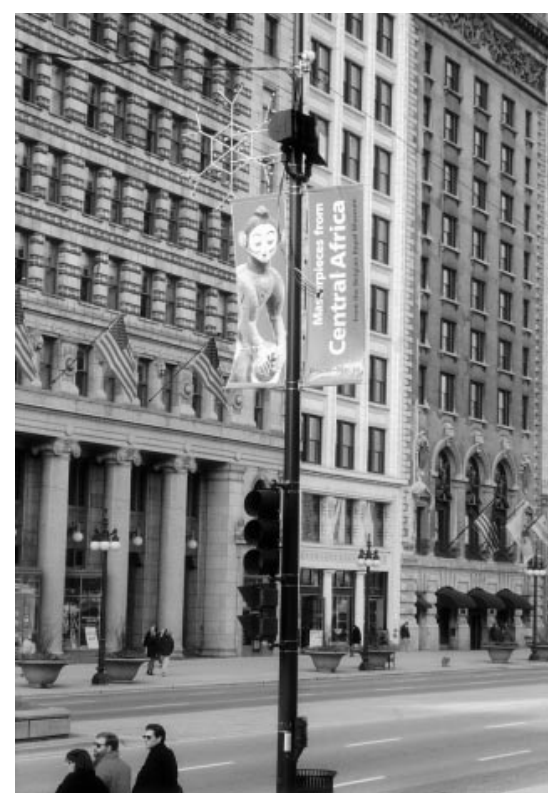

Foto 1 A Eine parallel zur Retroviruskonferenz laufende Ausstellung im Kunstmuseum von Chicago (Masterpieces from Central Africa) paßte dem Titel nach gut zum Eröffnungsvortrag über die Herkunft von HIV-1...

D Ähnlichkeit in der Genomanordnung zwischen SIVs und HIVs

- Stammesgeschichtliche Verwandtschaft zwischen den beiden Virusfamilien

D Prävalenz von SIVs in natürlichen Wirtsspezies

- Geographische Überschneidung der Verbreitungsgebiete

- Plausible Übertragungsmöglichkeiten.
Dr.Ulrich Marcus

Robert Koch-Institut, Nordufer 20, D-13353 Berlin 
Mit diesen Argumentationslinien läßt sich die Herkunft des HIV-2 von SIV $_{S M}$, einem bei in Westafrika verbreiteten Halsband-Mangaben vorkommenden SIV, belegen.

Angreifbarer war in dieser Hinsicht die Theorie, daß HIV-1 vom Schimpansen stammen müsse. Zwar sind die Schimpansen-SIVs dem HIV-1 nahe verwandt, es gibt jedoch bislang nur wenige, untereinander relativ weit auseinanderliegende SIV-Isolate von Schimpansen.

\section{Zu den neuen Erkenntnissen bezüglich der Herkunft von HIV-1 zählen:}

D Entdeckung von HIV-1-Subtyp N, welcher näher als alle anderen bekannten HIV-1-Subtypen mit SchimpansenSIVs verwandt ist

D Isolation eines weiteren SIV aus eingefrorenen Proben einer in einem amerikanischen Primatenzentrum 1985 verstorbenen Schimpansin [2]. Diese war Anfang der sechziger Jahre als Kind in einem unbekannten afrikanischen Land gefangen und nach Amerika gebracht worden. Sie war niemals für HIV-Forschung verwendet worden und hatte nach 1969 kein vom Menschen stammendes Material erhalten, welches HIV-Viren hätte enthalten können. 1985 waren bei einem Screening HIV-Antikörper bei ihr entdeckt worden. Die Schimpansin starb an Schwangerschafts- bzw. Entbindungskomplikationen, die mit der Lentivirusinfektion nicht in Zusammenhang standen. Das bei ihr isolierte SIV weist Verwandtschaftsbeziehungen zu Schimpansen-SIVs von zwei in Gabun gefangenen Schimpansen auf.

D Untersuchungen der Schimpansensubspezies der bisher bekannten SIVTräger ergaben, daß das am meisten divergente SIV-Isolat von einem Schimpansen der Subspezies "Schweinfurthii“ stammt, während die übrigen, näher verwandten Isolate alle aus Tieren der Subspezies ,Troglodytes" isoliert wurden. Das Verbreitungsgebiet der Troglodytes-Schimpansen umfaßt das westliche Zentralafrika (Kamerun, Äquatorial Guinea,
Gabun, Zentralafrikan. Republik, Volksrepublik Kongo und Kongo (Zaire) mithin die Region, in der die größte HIV-1-Subtypenvariabilität gefunden wird und aus der das erste nachweisbar HIV-positive menschliche Serum (aus dem Jahre 1959) stammt.

- Screening-Untersuchungen bei wildlebenden Schimpansen zeigen eine nicht unbeträchtliche SIV-Prävalenz. Im Unterschied dazu wird bei Schimpansen, die in Gefangenschaft und in Primatenzentren leben, bis auf Einzelfälle wie den oben beschriebenen, SIV nicht gefunden. Dies hängt damit zusammen, daß diese Schimpansen entweder schon in Gefangenschaft geboren und aufgezogen worden oder als Kinder in Gefangenschaft geraten sind. Falls SIV bei wildlebenden Schimpansen vorwiegend sexuell übertragen wird, müßte man aber zur Bestimmung der Virusprävalenz erwachsene Tiere untersuchen.

Die SIV-Variabilität zwischen verschiedenen Schimpansenspezies spricht für eine lange gemeinsame Evolution, d.h. das Virus kommt bei Schimpansen vermutlich bereits seit zehntausenden bis hunderttausenden von Jahren vor. Damit in Übereinstimmung steht die Beobachtung, daß nach gegenwärtiger Erkenntnis die SIV-Infektion für den Chimpansen nicht pathogen ist.
Virusübertragungen von Schimpansen auf den Menschen dürften im Laufe der Geschichte bereits wiederholt vorgekommen sein, da Schimpansen u.a. gejagt, geschlachtet und gegessen werden. In der Regel endeten solche Übertragungen früher wohl in epidemiologischen Sackgassen. Erst die Verbesserung der Verkehrswege, die zunehmende Erschließung des Urwalds durch den Menschen und die Zunahme der z. T. kommerziell betriebenen Jagd auf Schimpansen dürfte den Weg für eine HIV-Epidemie beim Menschen geebnet haben.

\section{Diagnostik, natürlicher Verlauf, Therapie und Prophylaxe der Primärinfektion mit HIV}

Vor allem seit von einigen Wissenschaftlern das Konzept „Hit hard and hit early" propagiert wird, wird vermehrt versucht, HIV-Infektionen noch früher, möglichst bereits im Stadium der ersten massiven Virusreplikation zu diagnostizieren und einer Behandlung zuzuführen. Da zu einem so frühen Zeitpunkt die Antikörperproduktion erst in Gang kommt, fallen ELISA- und/oder Western-Blot-Untersuchungen z.T. noch negativ oder fraglich aus. Im Rahmen einer Post-Expositionsprophylaxe-Studie in San Francisco wurde daher die Möglichkeit untersucht, einen quantitativen Virusgenomnachweis (HIV-PCR) für ei-

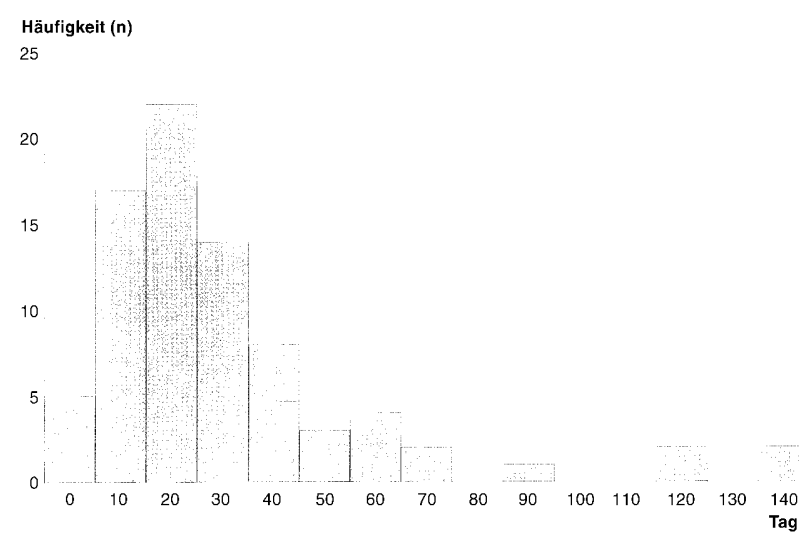

Abb. $1 \Delta$ Verteilung des Beginns der Symptomatik einer akuten retroviralen Infektion in Tagen nach dem vermutlichen Infektionsereignis bei einem Kollektiv von $\mathbf{8 0}$ frisch infizierten Patienten [nach Abstr. 573] 
ne möglichst frühzeitige Diagnostik einzusetzen [Abstr. 179]. Es zeigt sich, daß unter diesen Umständen (Screening von HIV-Ak-negativen HIV-exponierten Personen) mit einer nicht ganz unerheblichen Rate falsch positiver Ergebnisse zu rechnen ist (in einer Größenordnung von ca. $2 \%$ ). Die falsch positiven Resultate sind z.T. bei wiederholter Testung derselben Probe reproduzierbar. Die gemessenen Viruskonzentrationen sind in der Regel niedrig, d.h. unterhalb von 10 ooo Kopien/ml. Aufgrund dieser Beobachtung und der Erfahrung, daß die Mitteilung solcher Befunde an die exponierten Personen zu erheblichen psychischen Belastungen führt, empfehlen die Wissenschaftler aus San Francisco, einen quantitativen HIV-Genomnachweis nicht bei symptomlosen HIV-exponierten Personen zum Screening einzusetzen, da aufgrund der relativ niedrigen Übertragungswahrscheinlichkeiten ein positives Screening-Ergebnis in einer solchen Population in der Mehrheit der Fälle falsch positiv wäre. Auch von anderen Autoren werden derartige niedrig-falsch-positive Befunde berichtet [3].

Dabei ist durchaus nicht ganz auszuschließen, daß ein Teil dieser „falsch positiven " Befunde eigentlich gar nicht „falsch“ positiv ist. In Chicago wurde ein Untersuchung vorgestellt, in der erstmals beim Menschen von der Existenz einer latenten, seronegativen „HIV-Infektion" berichtet wird ([Abstr. 8] siehe auch Bgesbl.Ges.FS 3/99). In Affenmodellen ist eine solcher Zustand wiederholt beschrieben worden. Dabei kommt es zu einer transienten, schwach ausgeprägten Virämie nach einer Exposition, die jedoch nicht zur Serokonversion führt. Die Virusreplikation sistiert nach kurzer Zeit spontan und es finden sich im lymphatischen Gewebe latent virusinfizierte Zellen, eine Virusreplikation findet aber, wenn überhaupt, nur noch in minimalem Umfang statt und Infektiosität ist im Blut nicht nachweisbar. Mit Sicherheit gibt es aber auch wirklich „falsch positive“ Befunde, da z.T. auch Ausgangsuntersuchungen bei exponierten Personen unmittelbar (wenige Stunden) nach einer Exposition schon positiv ausfallen können.
Im Affenmodell kann sich in seltenen Fällen eine „latente“ seronegative Infektion spontan reaktivieren und dann zur Serokonversion und einer persistierenden produktiven Infektion führen. Ein ähnliches Phänomen könnte beim Menschen in den wenigen Fällen vorliegen, in denen zwischen Exposition und Serokonversion relativ lange Zeiträume liegen ( $>3$ Monate). Eine französische Untersuchung ging der Frage nach, wie lang die „Window-Periode" nach einer HIV-Exposition sein kann. Dafür wurden 80 im Rahmen der Serokonversion diagnostizierte HIV-Infizierte nach dem vermutlichen Infektionsereignis und -zeitpunkt befragt. 95\% der Befragten konnten ein wahrscheinliches Infektionsereignis innerhalb der vorangehenden drei Monate angeben mit dem Auftreten einer akuten klinischen Symptomatik im Mittel drei Wochen nach der Infektion, in vier Fällen wurden aber wahrscheinliche Infektionsereignisse angegeben, die vier bis fünf Monate zurücklagen (Abb. 1) [Abstr. 573].

Im Zusammenhang mit medikamentösen Postexpositionsprophylaxen (PEP) nach HIV-Exposition und frühzeitigem Behandlungsbeginn bereits in der Phase einer Primärinfektion sind noch folgende Beobachtungen und $\mathrm{Be}$ richte von Interesse:

D In einem Fall kam es nach einer beruflichen Exposition (Nadelstichverletzung) trotz rascher Einleitung (innerhalb einer Stunde) einer PEP mit vier Medikamenten (ZDV, ddI, 3TC, IDV), die sechs Wochen lang eingenommen wurden, zu einer HIV-Infektion. Die Infektionsquelle war ein zum Zeitpunkt des Unfalls mit ZDV+3TC behandelter HIV-positiver Drogengebraucher, welcher gleichzeitig mit HCV infiziert ist. Die HCV-Infektion wurde ebenfalls übertragen. Ein akutes retrovirales Syndrom trat bei der beruflich exponierten Person vier Wochen nach Ende der sechswöchigen Prophylaxe auf. Möglicherweise hängt das Versagen der PEP in diesem Fall mit bereits vorliegenden Resistenzen gegen $\mathrm{ZDV}$ und $3 \mathrm{TC}$ und/oder der gleichzeitigen HCV-Übertragung zu- sammen. Auch eine KombinationsPEP bietet also keine Gewähr für die Verhinderung einer HIV-Übertragung [Abstr. 210].

- Die meisten PEP-Regime bestehen entweder aus 2 NRTIs oder 2 NRTIs und 1 PI. Nach den italienischen Erfahrungen berichten unter Zweifachtherapie knapp 40\% der Behandelten über Nebenwirkungen, die bei ca. $10 \%$ zum vorzeitigen Prophylaxeabbruch Anlaß geben. Unter Tripeltherapie sind es $57 \%$ der Behandelten, die über Nebenwirkungen berichten, welche bei 22\% zum Abbruch der Prophylaxe führen. Zahlenmäßig ganz im Vordergrund stehen gastrointestinale $\mathrm{Be}$ schwerden, in einem Fall wird aber unter einer nichtberuflichen PEP mit einer Tripeltherapie die Manifestation einer Lipodystrophie beschrieben.

D Selbst bei hochmotivierten und intensiv betreuten Patienten, die eine HAART im Rahmen der Primärinfektion beginnen, ist die Abbruchrate erheblich. Etwa 50\% der am Aaron-Diamond-Institut in New York im Rahmen entsprechender Studien behandelten Patienten nehmen nach mehr als 24 Monaten ihre Medikamente immer noch [Abstr. 636]. Nach den bisherigen Erfahrungen stellt sich auch die Frage, ob eine vollständige Unterdrückung der Virusreplikation bei diesen Personen tatsächlich das anstrebenswerte Ziel darstellt: Probanden, bei denen es nicht $\mathrm{zu}$ einer durchgehenden dauerhaften Unterdrückung der Virusreplikation kam, sondern bei denen es kurze intermittierende Phasen von Virusvermehrung gab, entwickelten eine ausgeprägtere zelluläre Immunantwort gegen HIV als Probanden mit vollständiger Virussuppression.

\section{HIV-Subtypen}

Eine seit Jahren geführte Diskussion dreht sich um die Frage, ob es zwischen verschiedenen HIV-1-Subtypen Unterschiede bezüglich ihrer Infektiosität und/oder Pathogenität gibt. Bereits auf der internationalen AIDS-Konferenz von Genf/1998 wurden Beobachtungen einer Serokonverterstudie bei senegale- 
sischen Prostituierten präsentiert, die nun auch publiziert vorliegen [4]. In dieser Studie wird u.a. der Krankheitsverlauf bei verschiedenen Subtypen verglichen. Überblickt werden 53 Verläufe, davon 37 mit Subtyp A, jeweils fünf mit Subtyp C und D und sechs mit Subtyp G. Verglichen mit Subtyp A ermitteln die Autoren ein etwa achtfach höheres Erkrankungsrisiko für die Subtypen C und D (mit allerdings sehr breiten Konfidenzintervallen zwischen 1,22 und 57,80). Angesichts der niedrigen Fallzahlen müssen solche Schlußfolgerungen mit großer Vorsicht behandelt werden.

Größere Aussagefähigkeit haben hier sicherlich Studien, die den Krankheitsverlauf bei Subtyp B- und SubtypE-infizierten thailändischen Drogengebrauchern analysieren. In dieser Population, in der sich die beiden genannten HIV-1-Subtypen derzeit parallel ausbreiten, zeigen sich bisher keine Hinweise auf deutliche Pathogenitätsunterschiede zwischen B und E [Abstr. 711]. Interessanterweise werden aber im Rahmen der Primärinfektion im Durchschnitt innerhalb der ersten Monate bei Subtyp E-Infektion etwa doppelt so hohe Virustiter gemessen wie bei Subtyp B. Nach einem Jahr haben sich die Unterschiede aber eingeebnet. Höhere Plasma-Virustiter sind zwar noch kein Beweis für eine höhere Infektiosität, aber ein Hinweis, dem weiter nachgegangen werden sollte [Abstr. 275]. Ausgeprägte interindividuelle Unterschiede in der Viruslast gibt es aber auch ohne Subtypendifferenzen innerhalb des ersten Jahres nach einer Infektion. Erst etwa nach einem Jahr gibt das dann erreichte Level der Virusreplikation eine prognostisch relevante Information [5, Abstr. 273].

\section{Entwicklung der HIV-Epidemie in den USA}

Einen Einblick in die ungleiche geographische, soziale und ethnische Betroffenheit von der HIV/AIDS-Epidemie vermitteln die HIV-Inzidenzdaten bei USamerikanischen Blutspendern. Ebenso wie in Westeuropa werden Drogengebraucher und homosexuelle Männer in den USA dazu aufgefordert, kein Blut zu spenden. Die Seroinzidenzen bei Mehr- fachspendern spiegeln daher HIV-Neuinfektionen wider in einer Population, in der Personen mit offensichtlichen Infektionsrisiken bereits ausgesiebt wurden. Der landesweite Durchschnitt der HIVInzidenrate bei Mehrfachspendern lag in den Jahren 1993-1996 bei 9,6/100 ooo Personenjahren. Zwei- bis dreimal höhere Inzidenzraten als in den anderen $\mathrm{Al}$ tersgruppen werden bei den 25-44jährigen registriert und bei männlichen Spendern ist die Inzidenz doppelt so hoch wie bei weiblichen. Erhebliche Unterschiede gibt es in den verschiedenen geographischen Regionen: an der USWestküste und im Nordosten liegen die Seroinzidenzraten bei 1/100 ooo Personenjahren, im Südosten bei 25/100 ooo. Ein Nord-Südgefälle, wie es in Westeuropa beobachtet wird, gibt es offenbar auch in den USA. Noch auffälliger sind aber die Differenzen, wenn nach der ethnischen Herkunft der Blutspende differenziert wird: bei Afro-Amerikanern liegt die Inzidenzrate bei 51/100 ooo Personenjahren, bei Weißen nur bei 1/100 ooo [Abstr. 272]. Bemerkenswert ist, daß in den Regionen und Bevölkerungsgruppen, in denen erhöhte HIV-Inzidenzraten zu beobachten sind, auch andere Geschlechtskrankheiten in ähnlichem Aus$\mathrm{maß}$ stärker verbreitet sind.

Anlaß zur Sorge gibt in diesem $\mathrm{Zu}-$ sammenhang ein Anstieg der rektalen Gonorrhoe bei homosexuellen Männern in San Francisco seit 1994 auf fast das Doppelte [6]. Das Auftreten einer rektalen Gonorrhoe ist ein sicheres Indiz für vorangegangenen ungeschützten Analverkehr. Zeitgleich mit dem Anstieg der rektalen Gonorrhoe-Fälle zeigen Befragungen einen Rückgang des konsequenten Kondomgebrauchs und eine Zunahme von ungeschütztem Analverkehr. Ebenfalls beunruhigend ist, daß bei jungen, 15-22jährigen Homosexuellen aus New York, die erst in den vergangenen Jahren sexuell aktiv geworden sind, bereits $13 \%$ mit HIV infiziert sind [Abstr. 473, 474]. Die Hoffnung, der verbreitete Einsatz der neuen Kombinationstherapien könnte auch zu einem Rückgang der Neuinfektionen führen, hat sich in den USA bislang nicht realisiert [Abstr. S3o]. Bei der Anzahl der neudiagnostizierten HIV-Infektionen deutet nichts auf einen Rückgang hin. Dafür kommen mehrere Gründe in Frage:

- Ein großer Teil der Neuinfektionen durfte auf ungeschützte Kontakte mit relativ frischinfizierten Personen zurückzuführen sein, deren Infektion noch gar nicht diagnostiziert wurde und die daher auch nicht behandelt werden.

D Eine mäßige Verminderung der Infektiosität antiretroviral behandelter Personen könnte durch eine Zunahme von Risikoverhalten wieder konterkariert werden.

D Womöglich ist die Abnahme der Infektiosität genitaler Sekrete unter ART geringer, als durch den Therapie-bedingten Rückgang der Plasma-Viruslast suggeriert wird.

Die Tatsache, daß bei ca. $5-10 \%$ der Neuinfektionen Virusvarianten gefunden werden, die einzelne oder mehrere Medikamenten-induzierte Resistenzmutationen aufweisen, spricht dafür, daß zumindest ein Teil der HIV-Neuinfektionen durch Kontakte mit bereits als HIVpositiv diagnostizierten und ärztlich behandelten Personen erworben wird [Abstr. 217, 218, 125, 277, LB9]. Da nach derzeitigem Kenntnisstand resistente Virusvarianten eher und häufiger im Plasma als in Genitalsekreten nachweisbar sind, resistente Mutanten z.T. weniger effektiv übertragen werden als WildtypViren und Resistenzen z.T. im neuen Wirt in Abwesenheit eines medikamentösen Selektionsdruckes spontan revertieren, ist der Anteil der Neuinfektionen, der auf ungeschützten Verkehr mit bekannt HIV-Positiven zurückgeht, wahrscheinlich höher als $10 \%$.

Es gibt also keinen Anlaß, angesichts verbesserter Therapiemöglichkeiten die HIV-Primärprävention zu vernachlässigen - im Gegenteil, angesichts der Gefahr, daß resistente und multiresistente Viren übertragen werden könnten, muß insbesondere auch bei antiretroviral Therapierten dem Eindruck entgegengewirkt werden, das HIV-Übertragungsrisiko unter Therapie sei vernachlässigbar. Allerdings sind die bisher vorliegenden Untersuchungen zur Häufigkeit der Übertragung von Resistenzmutanten noch wenig repräsentativ und interpre- 
tationsbedürftig. Die meisten Studien suchen nach genotypischen Resistenzmutationen und machen keine Aussagen über das Ausmaß der phänotypischen Resistenz. Vor allem bei den sog. sekundären PI-Resistenzmutationen kann dies zu Fehlinterpretationen führen. Viele dieser sog. sekundären PI-Resistenzmutationen kommen als genetische Polymorphismen auch bei nicht PI-erfahrenen Viren vor, es handelt sich also häufig nicht um behandlungsinduzierte Mutationen. Auffällig war in einer der Untersuchungen eine relativ hohe Rate von NNRTI-Resistenzmutationen mit einer Clusterung im kalifornischen San Diego [Abstr. LB10]. Es wurde die Vermutung geäußert, daß dies mit mehreren Delavirdin-Monotherapie-Studien zusammenhängen könnte, die in San Diego im Verlauf der letzten Jahre durchgeführt wurden. Monotherapien mit NNRTI führen besonders schnell zur Resistenzbildung.

Weitgehend unklar sind noch die klinischen Konsequenzen einer Übertragung resistenter Virusvarianten. Bei einigen „Fitneß-mindernden“ Resistenzmutationen kann es zur Reversion zum Wild-Genotyp kommen. Inwiefern der resistente Genotyp „archiviert“ wird, und damit den klinischen Nutzen einer Behandlung mit entsprechenden Medikamenten vermindert, bleibt $\mathrm{zu}$ klären.

\section{Mutter-Kind-Übertragung}

Auf der Retroviruskonferenz in Chicago wurden die ersten Zwischenergebnisse der von UNAIDS koordinierten internationalen PETRA-Studie bekannt gegeben [Abstr. 57]. In der vierarmigen, in mehreren afrikanischen Ländern laufenden Studie werden drei Therapieschemata zur Prophylaxe der MutterKind-Übertragung mit einem PlazeboArm verglichen. Die Therapieschemata bestehen aus der Gabe von $\mathrm{ZDV}+3 \mathrm{TC}$ :

D Arm A: ab der 36. SSW, unter der Geburt und in der ersten Woche nach der Geburt (Dosis 4 mg/kg ZDV 2×/Tag, 2 mg/kg 3TC 2×/Tag)

D Arm B: unter der Geburt und in der ersten Lebenswoche

D Arm C: ausschließlich unter der Geburt.
Die an der Studie teilnehmenden Frauen stillen ihre Kinder zu zwei Drittel, ein Drittel (vorwiegend in Südafrika) hat die Möglichkeit, auf künstliche Babynahrung auszuweichen. Die Zwischenergebnisse (HIV-Übertragungsrate sechs Wochen nach der Geburt siehe Tabelle 1) zeigen, daß sowohl prä- als auch postnatale Behandlung zum Erfolg der Prophylaxe beitragen und daß auch mit kurzen Therapieregimen eine deutliche Wirkung erzielt werden kann. Die Studie bestätigt ähnliche Ergebnisse einer thailändischen Untersuchung, in der mit einem kurzen prä- und postnatalen ZDV-Regime vergleichbare Senkungen der Übertragungsrate erreicht wurden. Es ist unklar, ob die Kombination von ZDV mit 3TC zu einer Wirksamkeitssteigerung führt. In einer französischen Studie, in der eine ZDV+3TC-Prophylaxe ab der 32. SSW bis sechs Wochen nach Geburt mit der Standard ZDV-Prophylaxe (ab 14. SSW bis sechs Wochen nach Geburt) verglichen wurde, lag zwar die Übertragungsrate unter ZDV+3TC-Prophylaxe nochmals niedriger als unter ZDV-Prophylaxe (2,6\% vs. $6,5 \%)$, aber von den $\mathrm{ZDV}+3 \mathrm{TC}$-exponierten Kindern starben zwei im Alter von 11 und 13 Monaten an einer seltenen Funktionsstörung der Mitochondrien (durchschnittliche Häufigkeit 1:5000-1:20 000), was den Verdacht erweckt, daß es sich dabei um eine Nebenwirkung der pränatalen Nukleosidanaloga-Exposition handeln könnte [Abstr. 267].

Ein weiterer Nachteil der Kombinationsprophylaxe mit 3 TC ist das Auftreten von 3TC-Resistenzen (bei 39\% der behandelten Mütter). In Deutschland wird zur Prophylaxe der Mutter-KindÜbertragung eine ZDV-Monoprophylaxe ab der 32. SSW in Verbindung mit einer Kaiserschnittentbindung vor Einsetzen der Wehentätigkeit empfohlen, sofern nicht aus mütterlicher Indikation eine antiretrovirale Kombinationstherapie erforderlich ist. Mit diesem Vorgehen wird eine Übertragungsrate von unter $2 \%$ erreicht. Zusätzliche Vorteile bei einer kurzen Dauer der ZDV-Prophylaxe sind das verminderte Risiko einer Resistenzentwicklung gegen ZDV und ggf. einer Übertragung solcher resistenter Viren auf das Kind, wie sie unter

\begin{tabular}{|c|c|c|}
\hline & & $\%$ infizierte Kinder \\
\hline Arm A & $n=356$ & 8,6 \\
\hline Arm B & $n=343$ & 10,8 \\
\hline Arm C & $n=351$ & 17,7 \\
\hline Plazebo & $n=273$ & 17,2 \\
\hline
\end{tabular}

längeren Prophylaxeschemata beobachtet wird [Abstr. 265], sowie eine Verminderung des Risikos potentieller Schäden für die Kinder durch die intrauterine Exposition gegenüber Nukleosidanaloga.

Ein erhöhtes Risiko für Entwicklungsstörungen oder Langzeitschäden kann bei allein gegenüber ZDV exponierten Kindern $(n=230)$ nach einer Beobachtungsdauer von durchschnittlich vier Jahren glücklicherweise nicht festgestellt werden [7], aber eine weitere Langzeitbeobachtung und vor allem Verlaufsbeobachtungen bei Kindern, die in utero gegenüber antiretroviralen Kombinationstherapien exponiert waren, ist zweifelsohne notwendig.

Berichtet wurden Erfahrungen mit 89 Schwangerschaften unter PI-Kombinationstherapien sowie 53 Schwangerschaften unter PI- und/oder NNRTI(Nevirapin)-Kombinationstherapien. Unter den 89 Schwangerschaften waren 30 , bei denen die Kombinationstherapien auch im 1. Schwangerschaftsdrittel eingenommen wurden. Die Übertragungsrate liegt bislang bei o\%. Folgende Nebenwirkungen oder Geburtsschäden wurden berichtet, die möglicherweise mit der Medikamentenexposition zusammenhängen:

D 10 Kinder hatten eine Anämie

D ${ }_{5}$ Kinder hatten erhöhte Bilirubinwerte

D 3 Kinder hatten Durchfälle

D 2 Kinder hatten eine Neutropenie

D jeweils ein Kind hatte erhöhte Kreatininwerte, ein Down-Syndrom, beidseitige Brustgewebshypertrophie, Herzgeräusche, eine Inguinalhernie, einen kongenitalen Torticollis [Abstr. 686]. 
In der Serie mit 53 Schwangerschaften wurde bislang ebenfalls keine HIVÜbertragung festgestellt. An möglicherweise Medikamenten-bedingten Nebenwirkungen bei den Neugeborenen werden berichtet

D ein Down-Syndrom

D ein Vertikelseptumdefekt

D eine familiäre Syndaktylie

D eine Aplasia cutis [Abstr. 687].

In den USA sind nach den Ergebnissen einer vier Bundesstaaten umfassenden Studie etwa $80 \%$ der HIV-positiven Schwangerschaften bereits im frühen Verlauf der Schwangerschaft bekannt, etwa $20 \%$ der HIV-positiven Schwangeren gelangen aber erst sehr spät im Verlauf der Schwangerschaft in ärztliche Betreuung [Abstr. S6]. In Berlin waren 1996 ebenfalls etwa 80\% der Schwangerschaften HIV-positiver Mütter bekannt. Ob den nicht bekannten HIV-positiven Müttern von ihren Gynäkologen kein HIV-Test angeboten wurde, die Frauen keine Schwangerschaftsvorsorgeuntersuchungen wahrnahmen bzw. keine ärztliche Betreuung erhielten oder es sich um Serokonversionen im Verlauf der Schwangerschaft bei zunächst HIVnegativ getesteten Schwangeren handelt, läßt sich beim gegenwärtigen Kenntnisstand nicht sagen. Wäre die Berliner Situation repräsentativ für ganz Deutschland, müßte derzeit mit weniger als fünf HIV-Infektionen bei Neugeborenen pro Jahr in Deutschland gerechnet werden.

\section{Impfung}

Attenuierte Lebendimpfstoffe zählen zu den wirksamsten Impfstoffen, die zur Verfügung stehen. Mit Lebendimpfstoffen ist auch der bislang wirkungsvollste Schutz vor einer Infektion mit pathogenen Immunodefizienzviren erreicht worden. Das Haupthindernis für die weitere Entwicklung und den Einsatz von Lebendimpfstoffen gegen HIV ist deren fragwürdige Sicherheit [28]. Die „Attenuierung " bisher entwickelter Impfstoffkandidaten erfolgte durch Deletionen in regulatorischen Genabschnitten, v.a. im nef-Bereich, zusätzlich aber auch im vprund LTR-Bereich. Durch diese Deletionen wird die Replikationsfähigkeit des Impfvirus im Vergleich zum Wildtypvirus vermindert. Unabhängig von der Replikationsfähigkeit für die Pathogenität verantwortliche Gensequenzen wurden beim HIV bislang nicht identifiziert und können somit auch nicht zur Konstruktion eines apathogenen Impfvirus entfernt werden (zum Verhältnis zwischen pathogenen, AIDS auslösenden Viren, einem sicheren und wirksamen Lebendimpfstoff und einem sicheren, aber unwirksamen Lebendimpstoff siehe Abb. 2). Solange eine „Abschwächung " nur auf einer verminderten Replikationsfähigkeit basiert, können besondere Wirtsfaktoren sowie replikationsverbessernde Mutationen des Impfvirus dessen Pathogenität wiederherstellen. Baba et al. [29] berichten, daß sechs von acht Makaken- kindern, die mit einem im nef-, vpr- und NRE-Bereich deletierten SIV "geimpft“ worden waren, trotz dieser „Abschwächung" an AIDS erkrankten. Auch die beiden überlebenden Affenkinder zeigen Anzeichen eines sich entwickelnden Immundefektes. Nach längerer Beobachtungsdauer weisen auch vier von $16 \mathrm{er}-$ wachsenen, mit diesem „Impfvirus“ infizierte Makaken eine chronische Virämie auf, die bei bisher zwei Tieren zur klinischen Erkrankung führte. Ähnliche Beobachtungen werden aus Studien in anderen Primatenzentren berichtet. Auch bei einigen Menschen, die mit nefdeletierten Virusvarianten infiziert werden, wird inzwischen nach langen, symptomlosen Infektionsverläufen ein allmählicher Anstieg der Viruslast und ein Rückgang der CD4-Zellzahlen beobachtet. Neben diesen Beobachtungen werfen diverse weitere Experimente mit ,attenuierten "Lebendimpfstoffen in Tiermodellen Schatten auf diese bisher experimentell erfolgreichste Impfstrategie.

Ein holländisches Team kultivierte ein um drei Genabschnitte in LTR-, vprund nef-Bereich erleichtertes HIV-1 in einer Langzeitgewebekultur, um dessen genetische Stabilität zu prüfen. Zwar wurden die Gendeletionen nicht wieder repariert, aber durch eine Verdoppelung eines anderen Genabschnitts erlangte das ,abgeschwächte“ HIV-1-Konstrukt seine Fitneß fast vollständig zurück [30].

Eine amerikanische Gruppe analysierte ein durch in-vivo-Passage aus ei-

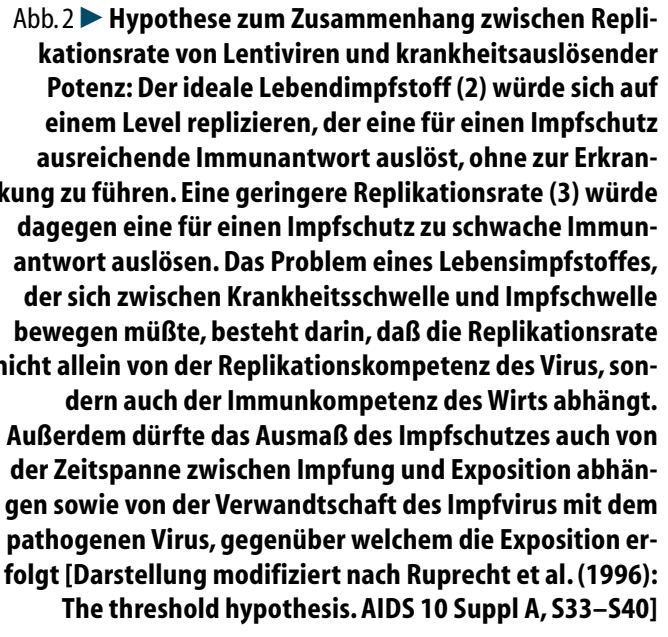

Abb. 2 Hypothese zum Zusammenhang zwischen Replikationsrate von Lentiviren und krankheitsauslösender Potenz: Der ideale Lebendimpfstoff (2) würde sich auf einem Level replizieren, der eine für einen Impfschutz ausreichende Immunantwort auslöst, ohne zur Erkrankung zu führen. Eine geringere Replikationsrate (3) würde dagegen eine für einen Impfschutz zu schwache Immunantwort auslösen. Das Problem eines Lebensimpfstoffes, der sich zwischen Krankheitsschwelle und Impfschwelle bewegen müßte, besteht darin, daß die Replikationsrate ( Außerdem dürfte das Ausmaß des Impfschutzes auch von der Zeitspanne zwischen Impfung und Exposition abhängen sowie von der Verwandtschaft des Impfvirus mit dem pathogenen Virus, gegenüber welchem die Exposition erThe threshold hypothesis. AIDS 10 Suppl A, S33-S40]

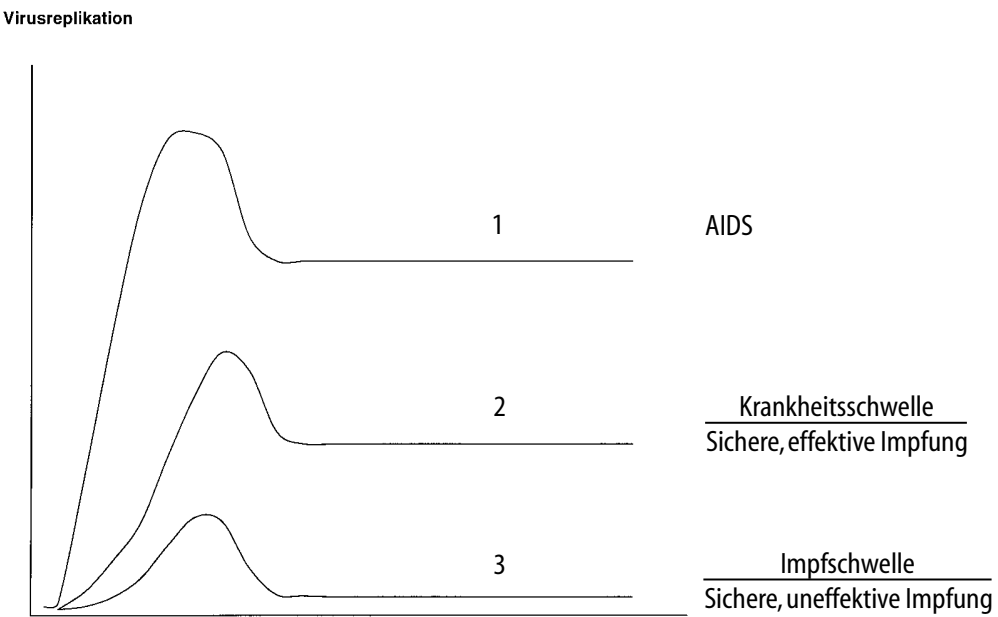


nem schwach replizierenden, apathogenen SHIV entstandenes gut replizierendes hochpathogenes SHIV. Der Unterschied, der dem Virus zu pathogenen Eigenschaften verhalf, lag lediglich in der Veränderung von zwölf GlykoproteinResiduen im Hüllbereich des Virus [31]. Ein anderes amerikanisches Team prüfte die Wirksamkeit zweier nef-deletierter SIV-Konstrukte als ,attenuierter“ Lebendvakzine. Alle fünf mit der weniger abgeschwächten Variante „immunisierten"Versuchstiere waren mit dem Challenge-Virus, einem SHIV-Konstrukt, infizierbar, zwei brachten es unter Kontrolle, drei erkrankten aber sogar schneller als die nicht-"immunisierten" Kontrolltiere. Das stärker „abgeschwächte“ Impfvirus zeigte ähnlich gemischte Resultate mit einem vollständigen Schutz, einem Impfversagen mit Erkrankung und drei Impfversagen mit zumindest vorläufiger Kontrolle des Challenge-Virus [32].

In einer anderen Studie mit nefund/oder vpr-deletierten Viruskonstrukten als Impfstoffen konnte nach subkutaner bzw. oraler „Immunisierung" von Versuchsaffen ebenfalls kein vollständiger Schutz vor einem vaginalen Viruschallenge erzielt werden. Zwei von zwölf immunisierten Versuchstieren entwickelten eine persistierende Infektion des Challenge-Virus, eines starb an AIDS. Bei den übrigen zehn Tieren war das Virus zwar im Plasma nicht nachweisbar, der Nachweis im Lymphknoten gelang aber [33].

Dittmer et al. untersuchte grundlegende Mechanismen protektiver Immunität gegenüber Retroviren in dem Modellsystem der Friend Virus-Infektion bei Mäusen [34]. Dieses Modell hat den Nachteil, daß es sich um ein dem HIV nicht verwandtes Onkovirus handelt, sein Vorteil besteht darin, daß die Bedeutung einzelner Komponenten des Immunsystems durch adoptive Transferexperimente überprüft werden kann, was in Primatenmodellen bislang nicht möglich ist. Die Wissenschaftler untersuchten die Bedeutung von CD4-positiven und CD8-positiven T-Lymphozyten sowie von B-Lymphozyten für eine protektive Immunität. Der wirksamste Schutz wurde durch eine Kombination aller drei Zellarten erzielt. Kombinatio- nen von nur zwei Zellarten konnten ebenfalls Schutz vermitteln, es waren aber deutlich höhere Zellzahlen erforderlich. Sofern die Ergebnisse aus diesem Modellsystem auf die Situation beim Menschen übertragbar sind, würden sie für Impfstrategien sprechen, die sowohl eine zelluläre als auch eine humorale Immunantwort induzieren.

Während die Bedeutung zellulärer Immunmechanismen für die Kontrolle und den Schutz vor einer HIV-Infektion inzwischen allgemein akzeptiert wird, ist die Bedeutung, die Antikörper beim Schutz vor einer HIV-Infektion spielen können, umstritten. In mehreren Tiermodellsystemen konnte mit neutralisierenden Antikörpern ein Schutz vor Lentivirusinfektionen oder eine Verminderung der anfänglichen Virusreplikation

\section{"Solange angesichts gravierender Sicherheitsbedenken an den Einsatz eines Lebendimpfstoffs beim Menschen nicht zu denken ist, müssen sich die Bemühungen darauf konzentrieren, die protektiv wirk- samen Immunmechanismen zu identifizieren und weniger riskante Impfstoffe zu konstruieren, die dieselben Immunmechanismen induzieren können."}

und eine Herabsetzung des Virus-Setpoints erreicht werden. Shibata et al. berichten, daß sie mit hochpotenten neutralisierenden Antikörpern gegen gp120 von HIV-1 Makaken vor einer SHIV-Infektion mit einer Viruschimäre mit einem HIV-1 Hüllprotein schützen konnten [35]. Die bei diesem Experiment verwendeten, von Schimpansen gebildeten anti-gp12o-Antikörper waren im Neutralisationstest bis zu tausendmal potenter als die meisten vom Menschen gegen HIV-1 gebildeten Antikörper und zeigten leider eine nur geringe Neutralisationsbreite, d.h. sie sind wirksam nur gegen die autologe Virusvariante und haben nur schwache kreuzneutralisierende Eigenschaften.

Weitere Experimente mit diesem Antikörper erbrachten, daß in seiner Anwesenheit die Clearance von freien
Viruspartikeln im Plasma deutlich schneller erfolgt [36]. Die Halbwertzeiten freier Viruspartikel werden durch den Antikörper von Werten zwischen 13 und 26 min auf Werte zwischen 4 und 7 min gedrittelt. Theoretisch könnten Antikörper also durchaus eine wichtige Rolle beim Schutz und bei der Bekämpfung der HIV-Infektionen spielen - das Problem ist, daß so wirksame und vor allem breiter wirksame Antikörper beim Menschen kaum gebildet werden [37]. In dieser Beziehung lassen Untersuchungen von LaCasse, Nunberg et al. aufhorchen $[38,39]$. Die geringe Neutralisationsfähigkeit von Antikörpern gegen die Virushülle von HIV wird v.a. darauf zurückgeführt, daß entscheidende Epitope nur transient im Rahmen der durch die Hüllprotein-CD4-Korezeptor-Bindung ausgelösten Konformationsänderung der Virushülle exponiert werden. Den Wissenschaftlern gelang das Kunststück, diese Übergangskonformation „einzufrieren“ und als Immunogen einzusetzen. Die von Mäusen dagegen gebildeten Antikörper besaßen eine breite Neutralisationsfähigkeit gegenüber einer Reihe primärer Isolate unterschiedlicher HIV-1-Subtypen und unterschiedlicher geographischer Herkunft.

\section{Kaposi-Sarkom}

Das Ende des vergangenen Jahrhunderts von dem österreichischen Arzt Moritz Kaposi erstmals beschriebene KaposiSarkom gibt den Wissenschaftlern auch heute noch Rätsel auf. So ist beispielsweise noch immer umstritten, ob es sich beim Kaposi-Sarkom um einen metastatisch wachsenden Tumor oder um einen chronisch-inflammatorischen Prozeß handelt, der zu einem nicht-malignem Wachstum hyperplastischer und hyperproliferierender Zellen führt. Bislang ist aus dem aus unterschiedlichen Zellen bestehenden Gewebe von Kaposi-Sarkomen noch keine autonom wachsende neoplastische Zelle isoliert worden und die als eigentliche Tumorzelle angesehene Spindelzelle ist nur manchmal monoklonalen, oft aber oligo- und sogar polyklonalen Ursprungs, was beides gegen einen primär malignen Tumor spricht [40]. 
Tabelle 2

Nachweis von HHV-8-DNS bei KS-Patienten (Quelle [35])

\begin{tabular}{lcc} 
& Nachweisrate & mittlere Zahl von Viruskopien/ $\mu$ g DS \\
\hline Leukozyten & $46 \%$ & 9000 \\
Plasma & $7 \%$ & 40 \\
Samenflüssigkeit & $12 \%$ & 300 \\
Speichel & $37 \%$ & 33000
\end{tabular}

\section{Man kennt vier epidemiologisch unter- scheidbare Formen des Kaposi-Sarkoms:}

D Das „klassische“ von Moritz Kaposi beschriebene Sarkom, welches vorwiegend bei älteren Männern aus der Mittelmeerregion und bei osteuropäischen Juden auftritt. Außer Alter und Geschlecht sind bei dieser Form keine sonstigen Kofaktoren bekannt.

D Das afrikanische Kaposi-Sarkom, das in allen Altersgruppen, bei Männern jedoch häufiger als bei Frauen auftritt. Kofaktoren sind nicht geklärt, da jedoch das Verbreitungsgebiet mit dem der Malaria übereinstimmt, wird eine Immunaktivierung durch diese oder andere chronische parasitäre Infektionen von einigen Wissenschaftlern als möglicher Kofaktor diskutiert.

D Das Kaposi-Sarkom bei Organtransplatierten. Bei diesen könnten die Immunsuppression und/oder die Zytokinaktivierung durch das Transplantat Kofaktoren darstellen.

D Das HIV-assoziierte Kaposi-Sarkom, bei dem ebenfalls der HIV-induzierte Immundefekt und/oder die Zytokinaktivierung durch HIV als Kofaktoren wirken könnten.

Das gemeinsame aller Kaposi-Formen ist der Nachweis einer HHV-8-Infektion. Die Gegenwart dieses neu entdeckten Herpesvirus ist zwar eine notwendige, aber nicht hinreichende Bedingung für das Entstehen eines Kaposi-Sarkoms. Bei der anhand der vorliegenden Untersuchungsergebnisse geschätzten Verbreitung von HHV-8 kommt auf ca. 17 ooo HHV-8-Infektionen nur eine Erkrankung an Kaposi-Sarkom. Offenbar sind also Kofaktoren notwendig, damit eine HHV-8-Infektion zur Entstehung eines Kaposi-Sarkoms führt. Der stärkste bekannte Kofaktor ist ohne Zweifel die Infektion mit HIV-1. Dabei erscheint es wenig wahrscheinlich, daß allein der HIV-induzierte Immundefekt die Kaposi-Entstehung auslöst. In-vitro-Studien weisen auf spezifischere Auswirkungen einer HIV-Infektion hin. Zum einen spielt eine Rolle die durch eine HIV-Infektion induzierte verstärkte Zytokinproduktion, wobei insbesondere die $\mathrm{Zy}$ tokine IFN- $\gamma$, TNF- $\alpha$, IL-1 und IL- 6 das Wachstum von Kaposi-Sarkomen stimulieren, zum anderen wirkt das tatProtein von HIV-1 Kaposi-stimulierend. Die tat-Wirkung wird auf eine besondere Struktur innerhalb des Proteins (das sog. RGD-Motiv) zurückgeführt, welche in den tat-Proteinen von HIV-2 und SIV nicht vorkommt. Bezeichnenderweise sind weder eine HIV-2-Infektion noch SIV-Infektionen bei Affen, die gleichzeitig mit HHV-8-verwandten Affen-Herpesviren infiziert sind, mit einer gesteigerten Kaposi-Sarkom-Erkrankungsrate verbunden.

Welche Rolle spielt nun das HHV-8 bei der Tumorentstehung? Ist HHV-8 selbst ein onkogenes, transformierendes Virus, welches direkt Zellen maligne entarten läßt? Die Beobachtung, daß die als Haupt-Tumorzellen angesehenen Spindelzellen gar kein HHV-8 enthalten, spricht nicht für diese Theorie. Eine alternative Erklärung wäre, daß vor allem eine gesteigerte HHV-8-Replikation ihrerseits wieder Zytokine wie z.B. VEGF induziert, die zur Zellhyperplasie führen. Aus dieser Zellhyperplasie könnten dann sekundär neoplastisch entartete Zellen entstehen. Die weitere Aufklärung der Tumorgenese ist keine aus- schließlich akademische Frage, da die unterschiedlichen Hypothesen auch unterschiedliche therapeutische Strategien implizieren. Falls die Zelltransformierung am Beginn des Geschehens steht, würde dies für den Einsatz von Zytostatika sprechen, falls zunächst eine erhöhte HHV-8-Replikation im Vordergrund steht, spräche dies für die Erprobung antiviraler Therapiestrategien (z.B. Foscarnet).

Zur Epidemiologie und den Übertragungswegen von $\mathrm{HHV}-8$ gibt es eine Reihe interessanter neuer Ergebnisse. Die retrospektive Untersuchung von Seren aus Kohortenstudien bei homosexuellen Männern in San Francisco und Amsterdam zeigt, daß es etwa zeitgleich mit der Ausbreitung von HIV-1 auch zu einer HHV-8-Epidemie unter schwulen Männern in den westlichen Metropolen kam [Abstr. 198]. In der Amsterdamer Kohortenstudie wurde eine gleichbleibende HHV-8-Inzidenz von 2,5\%/Jahr bei fallender HIV-1-Inzidenz (von 5,2\% auf 1,3\%/Jahr) registriert.

Das Risiko einer HHV-8-Infektion korrelierte dabei mit orogenitalen, nicht mit anogenitalen Kontakten. Interessant sind auch die Ergebnisse der Quantifizierung von HHV-8 in verschiedenen Geweben und Körperflüssigkeiten von KS-Patienten (Tabelle 2) [41]. Klar ist aus epidemiologischen Studien, daß HHV-8 sexuell übertragbar ist [42]. Der Weg der Übertragung ist aber unklar. Die in der Tabelle aufgeführte Viruslastdaten deuten darauf hin, daß Speichel bzw. orale Kontakte eine größere Rolle spielen könnten als bisher angenommen. Sie werfen aber auch Fragen auf: So gibt es beispielsweise trotz des relativ häufigen Nachweises von HHV-8-DNS in Leukozyten keinen Anhalt dafür, daß HHV-8 durch Blut übertragbar ist.

Vor allem in Afrika gibt es auch deutliche Hinweise auf die Möglichkeit einer Übertragung Mutter $\rightarrow$ Kind [43]. Auch dabei könnte Speichel natürlich eine Rolle spielen. An die Möglichkeit einer perinatalen oder intrapartalen Übertrag ist ebenfalls zu denken, zumal HHV-8-DNS auch in Zervixabstrichen HHV-8-infizierter Frauen nachweisbar ist [44]. 


\section{Literatur}

Die in [] aufgeführten Abstracts beziehen sich auf die der 6. Retroviruskonferenz 1999 in Chicago, welche auf der offiziellen Internet-Webseite der Konferenz http://www.retroconference.org zu finden sind

1. Weiss RA, Wrangham RW (1999) From Pan to pandemic. Nature 397:385-386

2. Gao F, Bailest E, Robertson DL, Chen Y, Rodenburg CM, Michael SF, Cummins LB, Arthur LO, Peeters M, Shaw GM, Sharp PM, Hahn BH (1999) Origin of HIV-1 in the chimpanzee Pan troglodytes troglodytes. Nature 397 : 436-441

3. Rich JD, Merriman NA, Mylonakis E, Greenough C, Flanigan TP, Mady BJ, Carpenter CJ (1999) Misdiagnosis of HIV infection by HIV-1 plasma viral load testing: a case series. Annals of Internal Medicine 130:37-39

4. Kanki PJ, Hamel DJ, Sankalé JL, Hsieh C, Thior I, Barin F, Woodcock SA, Guèye-Ndiaye A, Zhang E, Montano M, Siby T, Marlink R, Ndoye I, Essex ME, Mboup S (1999) Human immunodeficiency virus type 1 subtypes differ in disease progression. The Journal of Infectious Diseases 179:68-73

5. Kaufmann GR, Cunningham P, Kelleher AD, Zaunders J, Carr A, Vizzard J, Law M, Cooper DA, Sydney Primary HIV Infection Study Group (1998) Patterns of viral dynamics during primary human immunodeficiency virus type 1 infection. The Journal of Infectious Diseases 178: 1812-1815

6. Centers for Disease Control and Prevention CDC (1999) Increases in unsafe sex and rectal Gonorrhea among men who have sex with men - San Francisco, California, 1994-1997.MMWR 48:45-48

7. Culnane $M$, Fowler $M$, Lee $S S, M C$ Sherry $G$, Brady M, O'Donnell K, Mofenson L, Gortmaker SL, Shapiro DE, Scott G, Jimenez E, Moore EC, Diaz C, Flynn PM, Cunningham B, Oleske J (1999) Lack of long-term effects of in utero exposure to zidovudine among uninfected children born to HIV-infected women. JAMA 281:151-157

8. Farzadegan $\mathrm{H}$, Hoover, DR, Astemborski J, Lyles CM, Margolick JB, Markham RB, Quinn TC, Vlahov D (1998) Sex differences in HIV-1 viral load and progressiv to AIDS. Lancet 352:1510-1514

9. Moore RD, Cheever L, Keruly JC, Chaisson RE (1999) Lack of sex difference in CD4 to HIV-1 RNA viral load ratio. Lancet 353: 463-64
Kongressberichte

10. Junghans J, Ledergerber $B$, Chan $P$, Weber $R$, Egger M, Moroni $M$, on behalf of ICONA Study Group (1999) Sex differences in HIV-1 viral load and progression. (Correspondence) Lancet 353:589

11. Webber MP, Schoenbaum EE, Gourevitch MN Buono D, Klein RS (1999) A prospective study of HIV disease progression in female and male drug users. AIDS 13:257-262

12. Gallant JE, Chaisson RE, Keruly JC, Moore RD (1999) Stavudine in zidovudine (ZDV)experienced compared with ZDV-naive patients. AIDS 13:225-229

13. Lorenzi P, Opravil M, Hirschel B, Chave J-P, Furrer H-J, Sax H, Perneger TV, Perrin L, Kaiser L, Yerly S, Swiss HIV Cohort Study (1999) Impact of drug resistance mutations on virologic response to salvage therapy. AIDS 13: F17-F21

14. Padberg J, Schürman D, Grobusch $M$, Bergmann $F$ (1999) Drug interaction of isotretinoin and protease inhibitors: support for the cellular retinoic acid-binding protein-1 theory of lipodystrophy? (Correspondence) AIDS 13:284-85

15. Gervasoni C, Ridolfo AL, Trifirò G, Santambrogio S, Norbiato G, Musicco M, Clerici M, Galli M, Moroni M (1999) Redistribution of body fat in HIV-infected women undergoing combined antiretroviral therapy. AIDS 13 : 465-471

16. Tebas P, Patick AK, Kane EM, Klebert MK, Simpson JH, Erice A, Powderly W, Henry K (1999) Virologic responses to a ritonavirsaquinavir-containing regimen in patients who had previously failed nelfinavir. AIDS 13:F23-F28

17. Hellerstein M, Hanley MB, Cesar D, Siler S, Papageorgopoulos C, Wieder E, Schmidt D, Hoh R, Neese R, Macallan D, Deeks S, McCune JM (1999) Directly measured kinetics of circulating $\mathrm{T}$ lymphocytes in normal and HIV1-infected humans. Nature Medicine 5 : 83-89

18. Douek DC, McFarland RD, Keiser PH, Gage EA, Massey JM, Haynes BF, Polis MA, Haase AT, Feinberg MB, Sullivan JL, Janieson BD, Zack JA, Picker L, Koup RA (1998) Changes in thymic function with age and during the treatment of HIV infection. Nature 396:690-395

19. Nielsen SD, Ersbøll AK, Mathiesen L, Nielsen J0, Hansen JES (1998) Highly active antiretroviral therapy normalizes the function of progenitor cells in human immunodeficiency virus-infected patients. The Journal of Infectious Diseases 178:1299-1305

20. Merrill DP, Martinez-Picado J, Tremblay C, Sax PE, Boswell SL, Wong JT, D'Aquilla RT, Walker BD, Hirsch S (1999) Improved CD4 lymphocyte outgrowth in response to effective antiretroviral therapy. The Journal of Infectious Diseases 179: 345-351
21. Greenough TC, Sullivan JL, Desrosiers RC (1999) Declining CD4 T-Cell counts in a person infected with nef-delected HIV-1. (Correspondence) The New England Journal of Medicine 340:237

22. van Rompay KKA, Dailey PJ, Tarara RP, Canfield DR, Aguirre NL, Cherrington JM, Lamy PD, Bischofberger N, Pedersen NC, Marthas ML (1999) Early short-term 9-[2-(R)-(Phosphonomethoxy)Propyl] Adenine treatment favorably alters the subsequent disease course in simian immunodeficiency virus-infected newborn rhesus macaques. Journal of Virology 73:2947-2955

23. Schmitz JE, Kuroda MJ, Santra S, Sasseville VG, Simon MA, Lifton MA, Racz P, Tenner-Racz K, Dalesandro M, Scallon BJ, Ghrayeb J, Forman MA, Montefiori DC, Rieber EP, Letvin NL, Reimann KA (1999) Control of viremia in simian immunodeficiency virus infection by CD8+ lymphocytes. Science 283 : 857-860

24. Brodie SJ, Lewinsohn DA, Patterson BK, Jiyamapa D, Krieger J, Corey L, Greenberg PD, Riddell S (1999) In vivo migration and function of transferred HIV-1-specific cytotoxic T cells. Nature Med 5:34-41

25. Zajac AJ, Blattman JN, Murali-Krishna K, Sourdive DJD, Suresh M, Altman JD, Ahmed R (1998) Viral immune evasion due to persistence of activated T cells without effector function. J Exp Med188:2199-2204

26. Kalmas SA, Walker BD (1998) The critical need for CD4 help in maintaining effective cytotoxic $T$ lymphocyte responses. J Exp Med 188:2199-2204

27. Steger KK, Waterman PM, Pauza CD (1999) Acute effects of pathogenic simianhuman immunodeficiency virus challenge on vaccine-induced cellular and humoral immune responses to gag in rhesus macaques. Journal of Virology 73: 1853-1859

28. Johnson RP (1999) Live attenuated AIDS vaccines: hazards and drops. Nature Medicine 5:154-155

29. Baba TW, Liska V, Khimani AH, Ray NB, Dailey PJ, Penninck D, Bronson R, Greene MF, McClure HM, Martin LN, Ruprecht RM (1999) Live attenuated, multiple deleted simian immunodeficiency virus causes AIDS in infant and adult macaques. Nature Medicine 5: 194-203

30. Berkhout B, Verhoef K, van Wamel JLB, Back NKT (1999) Genetic instability of live, attenuated human immunodeficiendy virus type 1 vaccine strains. Journal of Virology 73 (2):1138-1145 
31. Cayabyab M, Karlsson GB, Etemad-Moghadam BA, Hofmann W, Steenbeke T, Halloran M, Fanton JW, Axthelm MK, Letvin NL, Sodroski JG (1999) Changes in human immunodeficiency virus type 1 envelope glycoproteins responsible for the pathogenicity of a multiply passaged simian-human immunodeficiency virus (SHIV-HXBC2). Journal of Virology 73 (2): 976-984

32. Lewis MG, Yalley-Ogunro J, Greenhouse J, Brennan TP, Bo Jiang J, VanCott TC, Lu Y, Eddy GA, Birx DL (1999) Limited protection from a pathogenic chimeric simian-human immunodeficiency virus challenge following immunization with attenuated simian immunodeficiency virus. Journal of Virology 73 (2): 1262-1270

33. Joag SV, Qian Liu Z, Stephens EB, Smith MS, Kumar A, Li Z, Wang C, Sheffer D, Jia F, Foresman L, Adany I, Lifson J, McClure HM, Narayan 0 (1998) Oral immunization of macaques with attenuated vaccine virus induces protection against vaginally transmitted AIDS. Journal of Virology 72 (11): 9069-9078
34. Dittmer U, Brooks DM, Hasenkrug KJ (1999) Requirement for multiple lymphocyte subsets in protection by a live attenuated vaccine against retroviral infection. Nature Medicine 5:189-193

35. Shibata R, Igarashi T, Haigwood N, BucklerWhite A, Ogert R, Ross W, Willey R, Cho MW, Martin MA (1999) Neutralization antibody directed against the HIV-1 envelope glycoprotein can completely block HIV1/SIV chimeric virus infections of macaque monkeys. Nature Medicine 5:204-216

36. Igarashi T, Brown C, Azadegan A, Haigwood N, Dimitrov D, Martin MA, Shibata R (1999)

Human immunodeficiency virus type 1 neutralizing antibodies accelerate clearance of cell-free virions from blood plasma. Nature Medicine 5:211-216

37. Moore JP, Burton DR (1999) HIV-1 neutralizing antibodies: How full is the bottle? Nature Medicine 5:142-144

38. LaCasse RA, Follis KE, Trahey M, Scarborough JD, Littman DR, Nunberg JH (1999) Fusioncompetent vaccines: Broad neutralization of primary isolates of HIV. Science 283 : 357-362

39. Montefiori DC, Moore JP (1999) Magic of the occult? Science 283:336-337

40. Gallo RC (1998) The enigmas of Kaposi's Sarcoma. Science 282:1837-1839
41. LaDuca JR, Love L, Abbott LZ, Dube S, Freidman-Kien AE, Poiesz BJ (1998) Detection of Human Herpesvirus 8 DNA sequences in tissues and bodily fluids. The Journal of Infectious Diseases 178:1610-1615

42. Blackbourn DJ, Osmond D, Levy JA, Lennette ET (1999) Increased Human Herpesvirus 8 seroprevalence in young homosexual men who have multiple sex contacts with different partners. The Journal of Infectious Diseases 179:237-239

43. He J, Bhat G, Kankasa C, Chintu C, Mitchell C, Duan W, Wood C (1998) Seroprevalence of Human Herpesvirus 8 among Zambian women of childbearing age without Kaposi's Sarcoma (KS) and mother-child pairs with KS. The Journal of Infectious Diseases 178: 1787-1790

44. Whitby D, Smith NA, Matthews S, O'Shea S, Sabin CA, Kulasegaram R, Boshoff C, Weiss RA, de Ruiter A, Best JM (1999) Human herpesvirus 8: seroepidemiology among women and detection in the genital tract of seropositive women. The Journal of Infectious Diseases 179:234-236 